Korean J Ophthalmol 2020;34(5):420-421 https://doi.org/10.3341/kjo.2020.0054

\section{Full-thickness Idiopathic Macular Hole in an Adolescent Male}

\section{Dear Editor,}

Idiopathic macular holes are typically observed in patients in the sixth or seventh decade of life. They are attributed to anomalous posterior vitreous detachment with vitreomacular traction, resulting in discontinuity of the neurosensory retina at the foveal center [1]. Macular holes in young patients are rare and are typically observed in the setting of blunt ocular trauma, with both tangential (caused by equatorial globe expansion) and anterior-posterior vitreomacular traction as causative factors. We present a case of full-thickness idiopathic macular hole in an adolescent male.

A 19-year-old engineering student presented to a tertiary emergency eye clinic with sudden loss of vision while driving. He denied any history of ocular trauma during initial and subsequent consultations with multiple interviewers. He also had no relevant medical history. His presenting visual acuity was $6 / 60$ in the right eye and $6 / 5$ in the left eye with no refractive correction. Examination of the anterior segment was unremarkable. Dilated fundus examination revealed a macular hole in the right eye. Optical coherence tomography (OCT) confirmed a full-thickness macular hole with a horizontal diameter of $502 \mu \mathrm{m}$ and no evidence of posterior vitreous detachment or epiretinal membrane (Fig. 1A). The left eye was normal. The patient was re-examined 4 weeks later, and no evidence of spontaneous closure was found; he subsequently consented to surgical repair via 25 -gauge pars plana vitrectomy.

Triamcinolone was used intraoperatively to stain the vitreous, which confirmed the posterior hyaloid was still attached at the posterior pole and peripheral retina. Posterior vitreous detachment was easily induced using the 25 -gauge vitrector on high vacuum. Brilliant blue G (ILM-Blue;

Received: April 20, 2020 Final revision: June 23, 2020

Accepted: June 29, 2020
Dutch Ophthalmic Research Centre, Zuidland, The Netherlands) dye was injected into the vitreous chamber revealing an area of incomplete staining with a radius of onedisc diameter from the foveal center, despite the absence of an overlying epiretinal membrane (Fig. 1B). Inner limiting membrane (ILM) peeling commenced outside the macula in the area of normal staining, before proceeding toward the fovea and extending peripherally to the vascular arcades. Following fluid-air exchange, 25\% sulphur hexalfluoride gas was injected into the vitreous cavity with the patient instructed to posture face down for 5 days. Hole closure was confirmed on OCT 2 weeks postoperatively (Fig. 1C). Best-corrected visual acuity of $6 / 18$ was achieved six months post-surgery with regeneration of the outer retinal layers observed on OCT (Fig. 1D).

Macular holes in young patients are rare and have been attributed to ocular trauma or pediatric retinal diseases [2]. Based on the absence of these precipitants, the pathogenesis of the macular hole in this adolescent is not clear. Intraoperative observations suggested both anterior-posterior and tangential traction were responsible. The posterior hyaloid was attached throughout the retina, but despite the patient's age, induction of posterior vitreous detachment was performed with ease. This would suggest diminution of vitreoretinal adhesion due to some prior event. An abnormal staining pattern was also noted around the macular
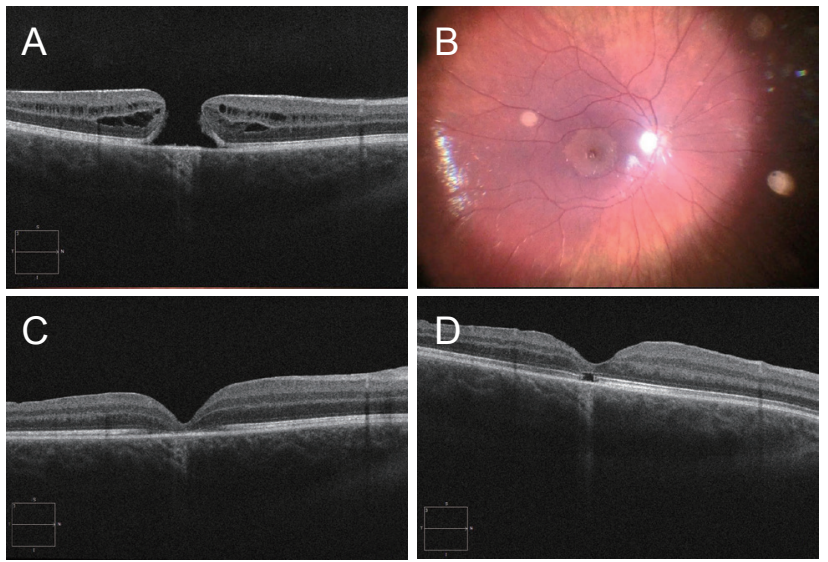

Fig. 1. Clinical images of (A) optical coherence tomography (OCT) at presentation, (B) intra operative image of non-staining, (C) OCT 2 weeks postoperatively, and (D) OCT 6 months postoperatively. 
hole. Brilliant blue $\mathrm{G}$ has been shown to have a high affinity for type IV collagen present on the ILM. A non-staining rim around macular holes has been reported previously; $39 \%$ of cases with a uniformly stained ILM were found to have incomplete staining around the macular hole [3]. Steel et al. [3] performed transmission electron microscopy in patients that had undergone macular hole surgery and found more vitreous surface tissue on the ILM in patients with an incomplete staining pattern. Furthermore, a central non-staining pattern was associated with greater hole diameter and lower post-operative visual acuity. The unstained rim was much larger in our case compared to the average size reported by Steel et al. [3] (1,500 vs. $291 \mu \mathrm{m})$. Residual cortical vitreous present on the ILM may have caused tangential traction at the macula.

Our case represents the first report of a full-thickness idiopathic macular hole in an adolescent patient. Previous pediatric cases have been reported in the literature $[2,4]$; however, all have involved children 10 years or younger, and congenital causes and non-accidental injuries were difficult to rule out. Our patient was legally autonomous and reported no potential causes of trauma in multiple interviews, thus eliminating trauma as the cause for the patient's presentation. Although small traumatic holes may experience spontaneous closure [5], this appeared unlikely in our case given the presenting hole size. Anatomical closure was achieved with a corresponding improvement in visual acuity.
Sebastian Derham, Riyaz Bhikoo

Department of Ophthalmology, Royal Perth Hospital, Perth, WA, Australia

E-mail (Sebastian Derham): sebastian.derham@health.wa.gov.au

\section{Conflict of Interest}

No potential conflict of interest relevant to this article was reported.

\section{References}

1. American Academy of Ophthalmology. Idiopathic macular hole preferred practice pattern. San Francisco: Elsevier; 2019. p. 189.

2. Shaikh N, Kumar V, Salunkhe N, et al. Pediatric idiopathic macular hole: a case report and review of literature. Indian J Ophthalmol 2020;68:241-4.

3. Steel DH, Dinah C, Madi HA, et al. The staining pattern of brilliant blue $\mathrm{G}$ during macular hole surgery: a clinicopathologic study. Invest Ophthalmol Vis Sci 2014;55:5924-31.

4. Lim LS, Fernandez-Sanz G, Levasseur S, et al. Idiopathic full thickness macular hole in a 10-year-old girl. Int $J$ Retina Vitreous 2018;4:25.

5. Liu W, Grzybowski A. Current management of traumatic macular holes. J Ophthalmol 2017;2017:1748135. 